C.Z. HUANG

KODAI MATH. J.

14 (1991), 113-122

\title{
REAL ZEROS OF SOLUTIONS OF SECOND ORDER LINEAR DIFFERENTIAL EQUATIONS
}

\author{
By CUN-ZHI HuANG
}

\section{Introduction}

We consider the second order linear differential equation

$$
f^{\prime \prime}+A f=0,
$$

where $A$ is an entire function. For an entire function $f$, let $\rho(f)$ be its order, $\mu(f)$ its lower order, $\lambda(f)$ the exponent of convergence of its zeros and $\lambda_{N R}(f)$ the exponent of convergence of its non-real zeros. In addition, we assume that the reader is familiar with the standard notation of Nevanlinna theory (see [4]).

When $A$ is a polynomial, the distribution of zeros of solutions of (1.1) has been studied extensively. The following theorem is well-known ([1]).

THEOREM A. If $A$ is a polynomial of degree $n \geqq 1$, then every solution $f \equiv 0$ of (1.1) satisfies

$$
\rho(f)=(n+2) / 2,
$$

and if $f_{1}, f_{2}$ are two linearly independent solutions of (1.1), then

$$
\lambda\left(f_{1} f_{2}\right)=(n+2) / 2 \text {. }
$$

Furthermore, G. Gundersen proved the following ([2]).

THEOREM B. Under the hypothesis of Theorem A,

$$
\lambda_{N R}\left(f_{1} f_{2}\right)=(n+2) / 2 .
$$

When $A$ is transcendental, we apply the lemma on the logarithmic derivative in Nevanlinna theory to (1.1) and can easily deduce that any solution $f \neq 0$ of (1.1) satisfies

$$
\rho(f)=+\infty \text {. }
$$

By analogy with Theorem A and Theorem B, we may hope that

* Supported by National Natural Science Foundation of P.R. China.

Received December 4, 1989; Revised July 5, 1990. 


$$
\lambda\left(f_{1} f_{2}\right)=+\infty
$$

or

$$
\lambda_{N R}\left(f_{1} f_{2}\right)=+\infty,
$$

where $f_{1}$ and $f_{2}$ are linearly independent solutions of (1.1). However, examples in [1] show that (1.6) and (1.7) may not hold if $\rho(A)$ is infinite or equal to a positive integer. When the growth of $A$ is suitably restricted, (1.6) and (1.7) hold.

Before stating the following results of J. Rossi, we make some definitions. Let $n_{+}(r, 1 / f)\left(n_{-}(r, 1 / f)\right)$ be the number of zeros of $f$ in $\{z:|z-(1 / 2) i r|$ $<(1 / 2) r\}(\{z:|z+(1 / 2) i r|<(1 / 2) r\})$, where $|z|>1$ and $r>0$. Define

$$
\lambda_{1}(f)=\varlimsup_{r \rightarrow \infty} \frac{\log \left(n_{+}(r, 1 / f)+n_{-}(r, 1 / f)\right)}{\log r} .
$$

Obviously $\lambda_{1}(f) \leqq \lambda_{N R}(f)$. The lower exponent of convergence $\lambda_{*}(f)$ of the zeros of an entire function $f$ is defined by

$$
\lambda_{*}(f)=\varliminf_{r \rightarrow \infty} \frac{\log n(r, 1 / f)}{\log r},
$$

where $n(r, 1 / f)$ is the number of zeros of $f$ in $|z|<r$.

J. Rossi proved the following ([8]).

THEOREM C. If $\rho(A) \leqq 1 / 2$ and $f_{1}, f_{2}$ are linearly independent solutions of (1.1), then

and

$$
\lambda_{1}\left(f_{1} f_{2}\right)=+\infty
$$

$$
\lambda_{*}\left(f_{1} f_{2}\right)=+\infty
$$

In this paper, we prove

THEOREM 1. Let $A$ be a transcendental entire function of order $\rho<+\infty$ with $k$ distinct finite asymptotic values. Suppose that $k=2 \rho$. If $f_{1}$ and $f_{2}$ are linearly independent solutions of (1.1), then

$$
\lambda_{1}\left(f_{1} f_{2}\right)=+\infty \text {. }
$$

THEOREM 2. Under the hypothesis of Theorem 1,

$$
\lambda_{*}\left(f_{1} f_{2}\right)=+\infty \text {. }
$$

\section{The Tsuji Characteristic}

In [9] (c.f. [6] and [7]) M. Tsuji introduced a characteristic for a function $f$ meromorphic in the upper half-plane based on the following Jensen-type formula : 


$$
\begin{aligned}
& \int_{1}^{r} \frac{n_{+}(t, 0)}{t^{2}} d t-\int_{1}^{r} \frac{n_{+}(t, \infty)}{t^{2}} d t \\
& =(2 \pi)^{-1} \int_{\sin ^{-1}\left(r^{-1}\right)}^{\pi-\sin ^{-1}\left(r^{-1)}\right.} \log \left|f\left(r(\sin \theta) e^{i \theta}\right)\right| \frac{d \theta}{r \sin ^{2} \theta}+O(1) .
\end{aligned}
$$

Here $n_{+}(t, 0)\left(n_{+}(t, \infty)\right)$ denotes the number of zeros (poles) of $f$ in $\{z:|z-(1 / 2) i t|$ $\leqq(1 / 2) t,|z| \geqq 1\}$.

He defined

$$
\begin{aligned}
& m_{+}(r, \infty)=m_{+}(r, f)=(2 \pi)^{-1} \int_{\sin ^{-1}(r-1)}^{\pi-\sin ^{-1(} r^{-1)}} \log ^{+}\left|f\left(r(\sin \theta) e^{i \theta}\right)\right| \frac{d \theta}{r \sin ^{2} \theta}, \\
& m_{+}(r, a)=m_{+}(r, 1 /(f-a)), \quad a \in C, \\
& N_{+}(r, \infty)=N_{+}(r, f)=\int_{1}^{r} \frac{n_{+}(t, \infty)}{t^{2}} d t=\sum_{1 \leqq r_{k} \leqq r \sin \phi_{k}}\left[\frac{\sin \phi_{k}}{r_{k}}-\frac{1}{r}\right],
\end{aligned}
$$

where $r_{k} e^{\imath \phi_{k}}$ are the poles of $f$ in $\operatorname{Im} z>0$,

$$
N_{+}(r, a)=N_{+}(r, 1 /(f-a)), \quad a \in C,
$$

and

$$
T_{+}(r, f)=m_{+}(r, f)+N_{+}(r, f) .
$$

For $f$ meromorphic in Im $z>0$, Tsuji proved the following properties.

(A) $m_{+}(r, a)+N_{+}(r, a)=T_{+}(r, f)+O(1), \quad a \in C$.

(B) If $f$ is also meromorphic in a neighborhood of the origin,

$$
m_{+}\left(r, f^{\prime} / f\right)=O\left(\log T_{+}(r, f)+\log r\right), \quad \text { n.e. }
$$

(C) $(q-2) T_{+}(r, f) \leqq \sum_{k=1}^{q} N_{+}\left(r, a_{k}\right)+O\left(\log T_{+}(r, f)+\log r\right)$ n.e. $\left(a_{k} \in C \cup\{\infty\}\right)$.

(n.e. means except on a set of finite linear measure.)

(D) $T_{+}(r, f)$ is a monotone increasing function of $r$. In $[7, \mathrm{p} .332]$ it is also proved that

(E) $\int_{R}^{\infty} \frac{m_{0, \pi}(r, f)}{r^{3}} d r \leqq \int_{R}^{\infty} \frac{m_{+}(r, f)}{r^{2}} d r \quad(R \geqq 1)$,

where

$$
m_{0, \pi}(r, f)=(2 \pi)^{-1} \int_{0}^{\pi} \log ^{+}\left|f\left(r e^{\imath \theta}\right)\right| d \theta
$$

Remark. Properties (A), (B) and (C) are analogues of Nevanlinna's first fundamental theorem, the lemma on the logarithmic derivative and Nevanlinna's second fundamental theorem, respectively.

Similarly, we can introduce the notations $T_{-}, m_{-}$and $N_{-}$for the lower halfplane analogues of the Tsuji functionals. 


\section{Preliminary Lemmas}

We need some lemmas.

LEMMA 1. Let $A$ be an entire function of order $\rho<+\infty$ with $k$ distinct finite asymptotic values $a_{\imath}(1 \leqq i \leqq k)$ and $L_{i}(1 \leqq i \leqq k)$ the asymptotic paths corresponding to $a_{\imath}$, which are simple curves from the origin to $\infty$ and non-intersecting except at the origin and divide the plane $\boldsymbol{C}$ into $k$ disjoint simply connected domains $D_{\imath}(1 \leqq i \leqq k)$. We may assume that $D_{\imath}$ is bounded by $L_{\imath}$ and $L_{\imath+1}(1 \leqq i \leqq k$; $\left.L_{k+1}=L_{1}\right)$. Suppose that $k=2 \rho$, then

(1) there exists in $D_{\imath}$ a path $\Gamma_{\imath}$ going to $\infty$ such that

$$
\lim _{\substack{|z| \rightarrow^{\infty} \\ z \in \Gamma_{\imath}}} \frac{\log \log |A(z)|}{\log |z|}=\rho,
$$

(2) $A(z)$ has no finite deficient values.

This lemma can be found in [10, p. 324 and p. 353].

LEMMA 2 [8]. Let $f$ be entire with infinite lower order such that

$$
\left.\begin{array}{l}
m_{+}(r, f)=O\left(r^{\alpha}\right) \\
m_{-}(r, f)=O\left(r^{\alpha}\right)
\end{array}\right\} \quad(r-\rightarrow \infty),
$$

where $0<\alpha<\infty$. Then, given $\lambda, 0<\lambda<\infty$,

$$
m(r, f)=(1+o(1))(2 \pi)^{-1} \int_{E(r)} \log ^{+}\left|f\left(r e^{2 \theta}\right)\right| d \theta, \quad \text { n.e. }
$$

where the angular measure is

$$
\operatorname{meas}(E(r))=O\left(r^{-\lambda}\right) .
$$

Applying Wiman-Valiron theory (c.f. [5]), we can deduce that

LEMMA 3. If $\rho(A)<\rho_{1}<\infty$ and $f$ is a solution of (1.1), then

$$
\log \log M(r, f) \leqq r^{\rho_{1}}, \quad\left(r \geqq r_{0}\right) .
$$

LEMMA 4. Let $\varepsilon>0$ be arbitrary and $E$ be entire. If there exist $\mu_{1}\left(0<\mu_{1}\right.$ $<\infty)$ and a sequence $R_{n} \rightarrow \infty$ such that

$$
\lim _{n \rightarrow \infty} \frac{\log M\left(R_{n}, E\right)}{R_{n}^{\mu_{1}}}=0,
$$

then

$$
\varlimsup_{n \rightarrow \infty}\left(\log R_{n}\right)^{-1} \int_{G(\varepsilon) \cap\left[1, R_{n}\right]} \frac{d r}{r} \leqq \varepsilon,
$$


where $G(\varepsilon)=\left\{r: \log M(2 r, E) \geqq r^{\mu_{1 / \varepsilon}}\right\}$.

(2) there exists a positive integer $q=q(\varepsilon)$ such that

$$
\left|\left(E^{\prime} / E\right)^{2}\left(r e^{i \theta}\right)-2\left(E^{\prime \prime} / E\right)\left(r e^{i \theta}\right)\right| \leqq r^{q} \quad \text { for } r \geqq r_{0}>1 \text {, }
$$

$r \bar{\in} G\left(\varepsilon^{2}\right)$ and $\theta \bar{\in} J_{r}$, where meas $\left(J_{r}\right) \leqq \varepsilon \pi$.

We remark that Lemma 4 is due to J. Rossi [8, Lemma 5 and 6]. But in his paper, he miswrote

$$
m\left(r,\left(E^{\prime} / E\right)^{2}-2 E^{\prime \prime} / E\right)=O(\log T(r, E)+\log r) \quad \text { for all } r \in \boldsymbol{R},
$$

it should be written as

$$
m\left(r,\left(E^{\prime} / E\right)^{2}-2 E^{\prime \prime} / E\right)=O(\log T(2 r, E)+\log r) \quad \text { for all } r \in \boldsymbol{R} .
$$

\section{Proof of Theorem 2}

Let $f_{1}, f_{2}$ be linearly independent solutions of (1.1). Set $E=f_{1} f_{2}$, and we note as in $[1]$ that

$$
-4 A=(c / E)^{2}-\left(E^{\prime} / E\right)^{2}+2\left(E^{\prime \prime} / E\right),
$$

where $c$ is the constant Wronskian of $f_{1}$ and $f_{2}$. Applying Nevanlinna theory to (4.1), we have

$$
T(r, E)=N(r, 1 / E)+\frac{1}{2} T(r, A)+O(\log T(r, E)+\log r), \quad \text { n.e. }
$$

Suppose that $\mu(E)<+\infty$, then there exist $\mu_{1}$ such that $\mu(E)<\mu_{1}<+\infty$, and $R_{n} \rightarrow \infty$ such that

$$
\lim _{n \rightarrow \infty} \frac{\log M\left(R_{n}, E\right)}{R_{n}^{\mu_{1}}}=0 .
$$

Fix $\varepsilon>0$. Since $A$ has no finite deficient values, it must have infinitely many zeros. Let $b_{1}, b_{2}, \cdots, b_{q+1}$ be $q+1$ zeros of $A$ with $q=q(\varepsilon)$ as in Lemma 4. Define

$$
H(z)=A(z) / \prod_{i=1}^{q+1}\left(z-b_{i}\right),
$$

then $H$ is entire and of order $\rho(H)=\rho(A)=k / 2$.

Set

$$
\begin{aligned}
& D(H)=\{z:|H(z)|>1\}, \\
& D(E)=\{z:|E(z)|>1\}, \\
& D\left(\varepsilon^{2}\right)=\left\{z=r e^{i \theta}: 0 \leqq \theta \leqq 2 \pi, r \bar{E} G\left(\varepsilon^{2}\right)\right\}, \\
& D=\left\{z=r e^{i \theta}: \theta \in J_{r}, r \bar{\in} G\left(\varepsilon^{2}\right)\right\},
\end{aligned}
$$


with $J_{r}, G\left(\varepsilon^{2}\right)$ as in Lemma 4.

From (3.8) and (4.1), we deduce that

$$
4|A(z)| \leqq|c|^{2}+|z|^{q}, \quad z \in D(E) \cap D\left(\varepsilon^{2}\right) \backslash D, \quad\left(|z| \geqq r_{0}\right) .
$$

But for $z \in D(H) \cap D\left(\varepsilon^{2}\right) \backslash D$,

$$
|A(z)|>\left(\frac{1}{2}|z|\right)^{q+1}, \quad\left(2|z| \geqq \max _{1 \leqq i \leqq q+1}\left|b_{i}\right|\right),
$$

From (4.4) and (4.5), we have for $r$ large enough $\left(r \geqq r_{*} \geqq r_{0}\right)$

$$
\left\{\theta: r e^{i \theta} \in D(H) \cap D(E) \frown D\left(\varepsilon^{2}\right)\right\} \cong J_{r} .
$$

Set

$$
L=\bigcup_{\imath=1}^{k} L_{\imath}
$$

with $L_{\imath}(1 \leqq i \leqq k)$ as in Lemma 1 . It is easy to see that

$$
D(H) \cap\{z:|z|>r\} \cap L=\phi,
$$

if $r$ is large enough. Without loss of generality, we may assume that $r=0$.

By Lemma 1 , there exists point $z_{i} \in D_{\imath}(1 \leqq i \leqq k)$ such that

$$
\left|H\left(z_{2}\right)\right|>e .
$$

Let $\Omega_{\imath}(1 \leqq i \leqq k)$ be the connected component of $D(H)$ containing the point $z_{\imath}$, then $\Omega_{i} \subset D_{\imath}(1 \leqq i \leqq k)$. By the maximum modulus principle, we conclude that $\Omega_{\imath}(1 \leqq i \leqq k)$ is unbounded.

Let

$$
r_{1}=\max \left\{r_{*},\left|z_{1}\right|,\left|z_{2}\right|, \cdots,\left|z_{k}\right|\right\}
$$

and $\theta_{i t}\left(1 \leqq i \leqq k ; r_{1} \leqq t<\infty\right)$ be the part of the circle $|z|=t$ in $\bar{\Omega}_{\imath}$ and $t \theta_{i}(t)$ the the linear measure of $\theta_{i t}$. We have

LEMMA 5.

$$
\varlimsup_{n \rightarrow \infty}\left(\log R_{n}\right)^{-1} \pi \int_{2 r_{1}}^{(1 / 2) R_{n}}\left(\sum_{\imath=1}^{k} \frac{1}{\theta_{i}(t)}\right) \frac{d t}{t}=\frac{k^{2}}{2}
$$

Proof. By a theorem of Tsuji [9], we have

(4.8) gives

$$
\begin{array}{r}
\log \left|H\left(z_{\imath}\right)\right| \leqq 9 \sqrt{2} \exp \left(-\pi \int_{2 r_{1}}^{(1 / 2) R n} \frac{d t}{t \theta_{i}(t)}\right) \log M\left(R_{n}, H\right) \\
\text { for } R_{n}>4 r_{1} \text { and } 1 \leqq i \leqq k
\end{array}
$$

$$
\pi \int_{2 r_{1}}^{(1 / 2) R_{n}}\left(\sum_{\imath=1}^{k} \frac{1}{\theta_{i}(t)}\right) \frac{d t}{t} \leqq k \log \log M\left(R_{n}, H\right)+k \log (9 \sqrt{2}) .
$$


Noting that

$$
k^{2} \leqq\left(\sum_{i=1}^{k} \theta_{i}(t)\right)\left(\sum_{i=1}^{k} \frac{1}{\theta_{i}(t)}\right) \leqq 2 \pi\left(\sum_{i=1}^{k} \frac{1}{\theta_{i}(t)}\right),
$$

from (4.9), we have

$$
\begin{aligned}
k^{2} / 2 \int_{2 r_{1}}^{(1 / 2) R_{n}} \frac{d t}{t} & \leqq \pi \int_{2 r_{1}}^{(1 / 2) R_{n}}\left(\sum_{\imath=1}^{k} \frac{1}{\theta_{i}(t)}\right) \frac{d t}{t} \\
& \leqq k \log \log M\left(R_{n}, H\right)+k \log (9 \sqrt{2}) .
\end{aligned}
$$

The desired conclusion follows from (4.11) and $\rho(H)=k / 2$.

Let

$$
\Delta(\varepsilon)=\left\{r: \sum_{i=1}^{k} \theta_{i}(r)<(2-\varepsilon) \pi\right\}
$$

and

$$
\beta=\varlimsup_{n \rightarrow \infty}\left(\log R_{n}\right)^{-1} \int_{\Delta(\varepsilon) \cap\left[2 r_{1},(1 / 2) R_{n}\right.} \frac{d t}{t},
$$

then we have

LEMMA $6 . \quad \beta=0$.

Proof. First we note that

$$
\begin{aligned}
& \pi \int_{2 r_{1}}^{(1 / 2) R_{n}}\left(\sum_{i=1}^{k} \frac{1}{\theta_{i}(t)}\right) \frac{d t}{t} \\
= & \pi \int_{\left.\Delta(\varepsilon) \cap\left[2 r_{1}, 1 / 2\right) R_{n}\right]}\left(\sum_{i=1}^{k} \frac{1}{\theta_{i}(t)}\right) \frac{d t}{t}+\pi \int_{\left[2 r_{1},(1 / 2) R_{n}\right]-\Delta(\varepsilon)}\left(\sum_{i=1}^{k} \frac{1}{\theta_{i}(t)}\right) \frac{d t}{t} \\
\geqq & \pi \int_{\Delta(\varepsilon) \cap\left[2 r_{1},(1 / 2) R_{n}\right]} \frac{k^{2}}{(2-\varepsilon) \pi} \frac{d t}{t}+\pi \int_{\left[2 r_{1},(1 / 2) R_{n}\right]-\Delta(\varepsilon)} \frac{k^{2}}{2 \pi} \frac{d t}{t} \\
= & \left(\frac{k^{2}}{2-\varepsilon}-\frac{k^{2}}{2}\right) \int_{\Delta(\varepsilon) \cap\left[2 r_{1},(1 / 2) R_{n}\right]} \frac{d t}{t}+\frac{k^{2}}{2} \int_{2 r_{1}}^{(1 / 2) R_{n}} \frac{d t}{t} .
\end{aligned}
$$

From (4.7) and (4.13), we have

$$
\frac{k^{2}}{2} \geqq \frac{k^{2}}{2}+\left(\frac{k^{2}}{2-\varepsilon}-\frac{k^{2}}{2}\right) \beta .
$$

We note that the right-hand side of (4.14) is strictly greater than $k^{2} / 2$ unless $\beta=0$. Hence $\beta=0$.

Let $\Omega(E)$ be a connected component of $D(E)$ and $\theta_{t}$ be the part of the circle $|z|=t$ in $\Omega(E)$ and $t \theta(t)$ the linear measure of $\theta_{t}$, then again by the theorem of Tsuji [9], we have 


$$
\begin{aligned}
& \log \log M\left(R_{n}, E\right) \geqq \pi \int_{2 r_{1}}^{(1 / 2) R_{n}} \frac{d t}{t \theta(t)} \\
\geqq & \pi \int_{\left[2 r_{1},(1 / 2) R_{n}\right]-G\left(\varepsilon^{2}\right)-\Delta(\varepsilon)} \frac{d t}{t \theta(t)} \\
\geqq & \pi \int_{\left[2 r_{1},(1 / 2) R_{n}\right]-G\left(\varepsilon^{2}\right)-\Delta(\varepsilon)} \frac{d t}{2 \varepsilon \pi t} \\
\geqq & \frac{1}{2 \varepsilon}\left(\int_{2 r_{1}}^{(1 / 2) R_{n}} \frac{d t}{t}-\int_{\left[2 r_{1}, R_{n}\right] \cap G\left(\varepsilon^{2}\right)} \frac{d t}{t}-\int_{\left[2 r_{1},(1 / 2) R_{n}\right] \cap \Delta(\varepsilon)} \frac{d t}{t}\right) .
\end{aligned}
$$

From (3.7), (4.12) and (4.15), we have

$$
\varliminf_{n \rightarrow \infty} \frac{\log \log M\left(R_{n}, E\right)}{\log R_{n}} \geqq \frac{1}{2 \varepsilon}\left(1-\varepsilon^{2}\right) .
$$

Since $\varepsilon$ is arbitrary, we can make the right-hand side of (4.16) larger than $\mu_{1}$, by choosing a small $\varepsilon$ at the beginning. This contradicts (4.3). Hence $\mu(E)=+\infty$.

For any $\alpha>1$, we have by (4.2)

$$
1 / 2 T(r, E) \leqq N(\alpha r, 1 / E)+1 / 2 T(\alpha r, A), \quad(r \text { large enough }) .
$$

We note that $\rho(A)<+\infty$, then (4.17) and $\mu(E)=+\infty$ give

$$
\varliminf_{r \rightarrow \infty} \frac{\log N(r, 1 / E)}{\log r}=+\infty,
$$

which implies $\lambda_{*}\left(f_{1} f_{2}\right)=+\infty$. Theorem 2 is proved.

\section{Proof of Theorem 1}

Properties (A) and (B) together with (4.1) give

$$
T_{+}(r, E)=N_{+}\left(r, \frac{1}{E}\right)+\frac{1}{2} T_{+}(r, A)+O\left(\log T_{+}(r, E)+\log r\right) \text {, n.e. }
$$

We assume that

$$
\lambda_{1}(E)<+\infty
$$

and will arrive at a contradiction from this assumption.

From (5.1) and (5.2), we have

similary

$$
T_{+}(r, E)=O\left(r^{\alpha}\right), \quad(0<\alpha<\infty),
$$

$$
T_{-}(r, E)=O\left(r^{\alpha}\right), \quad(0<\alpha<\infty) .
$$

Since $\rho(A)<+\infty$, we can choose $\lambda$ such that $\rho(A)<(1 / 2) \lambda<+\infty$. Theorem 2 gives that $\mu(E)=+\infty$. Applying Lemma 2 to $E$, we have 
REAL ZEROS OF SOLUTIONS OF DIFFERENTIAL EQUATIONS

$$
m(r, E)=O\left(r^{-\lambda} \log M(r, E)\right) \text { n.e. }
$$

By a theorem of Hayman and Stewart [3, Theorem 6], for any constant $K>1$, we have

where

$$
\log M(r, E) \leqq m(r, E)[\log m(r, E)]^{K}, \quad r \in G,
$$

$$
\varliminf_{r \rightarrow \infty}(\log R)^{-1} \int_{G_{\cap}[1, R]} \frac{d t}{t}>0 .
$$

From (5.5), (5.6), (5.7) and Lemma 3 with $\rho_{1}=(1 / 2) \lambda$, there exist a sequence $r_{n} \rightarrow \infty$ and a constant $c$ such that

$$
1 \leqq c r_{n}^{-\lambda}\left[\log \log M\left(r_{n}, E\right)-\lambda \log r_{n}+\log c\right]^{K} \leqq c r_{n}^{-\lambda+(1 / 2) K \lambda} .
$$

If we choose $K<2,(5.8)$ gives a contradiction. The proof of Theorem 1 is complete.

Remark. Indeed, we have proved the following slightly stronger results.

THEOREM 3. Let $A$ be the same as in Theorem 1 and $P$ a non-constant polynomial. If $f_{1}$ and $f_{2}$ are linearly independent solutions of the differential equation

$$
f^{\prime \prime}+A P f=0,
$$

then

$$
\lambda_{1}\left(f_{1} f_{2}\right)=+\infty .
$$

THEOREM 4. Under the hypothesis of Theorem 3,

$$
\lambda_{*}\left(f_{1} f_{2}\right)=+\infty \text {. }
$$

Acknowledgement. We would like to thank Professor Chong-ji Dai and Guodong Song for their help. We would also like to thank the referee for his careful reading of my manuscript and making some corrections.

\section{REFERENCES}

[1] S. BANK AND I. Laine, On the oscillation theory of $f^{\prime \prime}+A(z) f=0$ where $A(z)$ is entire, Trans. Amer. Math. Soc., 273 (1982), 351-363.

[2] G. Gundersen, On the real zeros of solutions of $f^{\prime \prime}+A f=0$ where $A$ is entire, Ann. Acad. Sci. Fenn., 11 (1986), 275-294.

[3] W.K. HAyman AND F. STEwart, Real inequalities with applications to function theory, Proc. Cambridge Philos. Soc., 50 (1954), 250-260.

[4] W.K. Hayman, Meromorphic functions, University Press, Oxford (1964).

[5] W.K. Hayman, The local growth of power series: A survey of the WimanValiron method, Canad. Math. Bull., 17 (1974), 317-358.

[6] S. Hellerstein AND C.C. YANG, Half-plane Tumura-Clunie theorems and the 
real zeros of successive derivatives, J. London Math. Soc. (2), 4 (1972), 469-481.

[7] B. JA. LEVIN AND I.V. Ostrovskil, On the dependence of the growth of an entire function on the distribution of the zeros of its derivatives, Amer. Math. Soc. Translations (2), 32 (1963), 323-357.

[8] J. Rossi, The Tsuji characteristic and real zeros of solutions of second order ordinary differential equations, J. London Math. Soc. (2), 36 (1987), 490-500.

[9] M. Tsujl, On Borel's directions of meromorphic functions of finite order. 1, Tôhoku Math. J., 2 (1950), 97-112.

[10] GuANG-HOU Zhang, Theory of entire and meromorphic functions, Science Press (Chinese) (1986).

Department of Mathematics

East China Normal University

SHANGHAI, 200062

P. R. CHINA 